\title{
Mycological Examination of Dust from Mouldy Hay Associated with Farmer's Lung Disease
}

\author{
BY P. H. GREGORY AND MAUREEN E. LACEY \\ Rothamsted Experimental Station, Harpenden, Hertfordshire
}

(Received 25 April 1962)

\begin{abstract}
SUMMARY
Small samples of hay were shaken in a perforated drum in a wind of $4.2 \mathrm{~m}$. $/ \mathrm{sec}$.; the liberated dust cloud was sampled with the cascade impactor for microscopical examination, and with the Andersen sampler for identification of organisms in culture. The results of testing batches of hay showed large differences in microbial content. Twenty-eight batches classed as 'good hay' gave up to 3 million spores/g. dry wt. hay, mainly Aspergillus glaucus with Cladosporium spp. and Hemispora stellata. Dust blown from seventeen batches of 'mouldy hay' differed greatly in composition and had from 5 to $\mathbf{2 5 0}$ million spores/g.: abundant forms included A. glaucus, A. fumigatus, A. nidulans, Penicillium spp., Absidia spp., Mucor pusillus, bacteria, many actinomycetes. Fourteen batches of mouldy hay associated with cases of 'farmer's lung' disease were generally similar in mould content to the batches of mouldy hay, but had many more spores of Humicola (Monotospora) lanuginosa and $\boldsymbol{A}$. fumigatus, and were specially characterized by very many actinomycetes. Farmer's lung hays tended to neutrality (averaging $\mathrm{pH} \mathbf{7 \cdot 0}$, as compared with $\mathrm{pH} 5$ to 6 of other hays), and were rich in thermophilic organisms, commonly with hundreds of millions of actinomycete spores/g., a large proportion of which grew readily at $60^{\circ}$. Hays associated with farmer's lung appear to have heated spontaneously to a higher temperature during maturation than the other hays. Dust from all hays contained particles of higher plants, but there were not obviously more in farmer's lung than in other hays. All types of spores were shown to be potentially able to penetrate to the deeper parts of the lung (especially the spores of actinomycetes, $A$. fumigatus and $\boldsymbol{H}$. stellata), but whether these organisms play any part in the aetiology of the disease is unknown.
\end{abstract}

\section{IN'TRODUCTION}

Farmer's lung is a disease attributed to inhalation of dust from mouldy hay. This disease (reviewed by Frank, 1958) has been known since 1932 (Fuller, 1958), but interest in it has increased recently, perhaps because of changes in haymaking practice caused by the pick-up baler. The aetiology of farmer's lung is still obscure and the purpose of our work was to assess the possible role of the microbial components of the inhaled dust from mouldy hay. Previous research on spoilage of stored hay has been concerned mainly with self-heating (Browne, 1929) and spontaneous combustion (Miehe, 1930). The development of the microbial flora on grass composts has been studied by Webley (1947), Forsyth \& Webley (1948) and by Eastwood (1948). Our work involved developing and testing of methods for sampling dust from hay, and examining farm hays (including both good hays and 
hays associated with human and animal disease). The microfloral succession in experimental batches of hay baled at different moisture contents will be described later.

\section{METHODS}

Farmer's lung disease is attributed to the inhalation of dust from hay by farm workers when feeding mouldy hay to cattle in cowsheds. Hays were therefore tested by shaking in air and taking samples of the air for examination. The standard method of shaking in water and plating out dilutions was considered inappropriate because it would remove many kinds of particles which remain firmly attached to dry hay and which do not normally enter the air-borne dust fraction. (The washing method was, however, used for comparison with the blowing method in early tests.)

The method adopted after preliminary trials (Gregory \& Lacey, 1963) uses reproducible conditions to give a standard test by which various batches of hay can be compared. Because the primary pathological changes occur in the deeper parts of the lung, it was considered useful to classify the dust into two fractions: (1) 'nonpenetrating' larger particles which would be deposited mainly in the upper respiratory tract, nose, throat, bronchi and larger bronchioles; (2) 'penetrating' smaller particles which would be expected to reach the alveoli. Particles aerodynamically similar to unit-density spheres $5 \mu$ diam. are considered to have a $50 \%$ chance of penetrating to the alveoli, and this particle diameter was accordingly chosen to separate the two fractions, although the limiting size depends to some extent on the activity of respiration (Davies, 1952).

Hay sampling. Hay for test was normally received from the farm as whole or part bales and stored under cover at outdoor air temperatures (slices from bales were stored in large unsealed polythene bags). Samples for test were removed from the stored hay by pulling a handful from near the centre of the bale and carried to the laboratory in a small polythene bag with as little shaking as possible. (A 5-g. subsample was cut into $3 \mathrm{~cm}$. lengths and occasionally stirred while in $250 \mathrm{ml}$. water for $15 \mathrm{~min}$. before the $\mathrm{pH}$ value was measured electrometrically.)

Shaking in air. A sample of about 20-30 g. hay was tipped from the polythene bag into a cylinder of perforated zinc $(2 \mathrm{~mm}$. diam. perforations) with Perspex ends, which was mounted horizontally to rotate on bearings across a wind-tunnel of square cross-section with $29 \mathrm{~cm}$. sides (Fig. 1). The cylinder was rotated during test at about $60 \mathrm{rev} . / \mathrm{min}$. by an electric motor to give a gentle 'tedding' action on the hay sample. Air was drawn down the wind-tunnel by a fan, a speed of about $4.2 \mathrm{~m}$./sec. being adopted in routine tests. The dust cloud blown out of the cylinder travelled with the wind and reached the sampling position after a diffusion path of $1.2 \mathrm{~m}$.

Air-sampling methods. A general study of hay dust must be based primarily on microscopic examination to reveal all its components, and must be supplemented by cultural examination for more precise identification of any organisms that can be grown in culture. For microscopic examination, air was sampled with a Casella model of the cascade impactor (May, 1945), connected to a vacuum pump drawing $20 \mathrm{l}$./min. through the orifice. Sampling at this rate was approximately isokinetic at the wind speed used. The cascade impactor accelerates the air-stream through four successively narrowing jets, impacting the entrained dust particles, graded into 
four size fractions, on glass microscope slides made sticky with glycerol jelly applied on strips of transparent cellulose film. After exposure the strips were removed from the slides and the four dust traces mounted on a single microscope slide in glycerol jelly before examination ( $\mathrm{Pl} . \mathrm{1})$. In routine tests with the cascade impactor the sticky slides often had to be changed after the first minute of shaking, to avoid overloading the slides. The band of dust, which consisted mainly of fungal and actinomycete spores, was scanned and counted under a $3.75 \mathrm{~mm}$. fluorite oilimmersion lens, the commoner types of particles being recorded on electrically operated counters. Recognition of the various types was facilitated by comparison with cultures and direct mounts from hay.

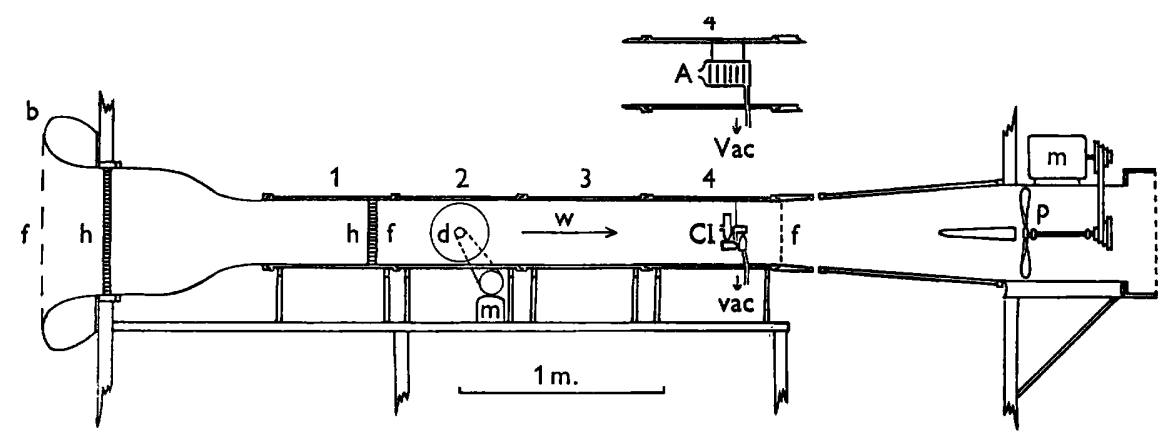

Fig. 1. Diagram of wind-tunnel showing position of sampling apparatus. 1-4, Perspex working sections ; $b$, bell-shaped intake; $h$, paper honeycomb; $f$, filter screens; $m$, electric motors; p, fan; d, perforated zinc cylinder; CI, cascade impactor; A, Andersen sampler; vac, to vacuum pump; $\mathbf{w}$, wind direction.

Calculation of numbers of spores blown from hay. Estimates of numbers of spores are given per g. dry wt. hay. Numbers of particles liberated per minute from a sample of hay were estimated as follows. The number of spores impacted from 20 l. air was first estimated by scanning the four dust traces from the cascade impactor. As $21.5 \mathrm{~m} .^{3} \mathrm{air} / \mathrm{min}$. flowed down the wind-tunnel, the number caught in the impactor was multiplied by $1075\left(=21.5 \mathrm{~m} .{ }^{3} / 20 \mathrm{l}\right.$.) and divided by the dry weight of the sample to give the total count/g. hay.

In the conditions used about $50 \%$ of unit density spheres of $4.5 \mu$ diam. would penetrate the first two jets of the cascade impactor, and $30 \%$ of particles $0.5 \mu$ diam. would be captured at the fourth stage. Accordingly particles captured behind jets 1 and 2 are classified as 'non-penetrating', and those captured behind jets 3 and 4 as 'penetrating', with respect to entry of the lung.

Preliminary tests of method. From results of preliminary tests with mouldy hay shaken in the wind-tunnel, a period of $3 \mathrm{~min}$. blowing at a wind speed of $4 \cdot 2 \mathrm{~m}$./ $/ \mathrm{sec}$. was chosen as the standard for routine testing (Gregory \& Lacey, 1963). Because conditions are not uniform within a bale of hay, the results from repeat tests differed. Visual counts of spores blown from successive samples from single bales of a good hay $(\mathrm{L})$ and of a hay associated with a case of farmer's lung (H 44) illustrate this variability (Table $\mathbf{1}$ ).

Cultural examination. In preliminary tests several methods of sampling for the culturable fraction were tested, including the use of alginate wool filters and the liquid impinger. Both these methods ultimately placed the organisms in suspension 
in water from which serial dilutions can be plated. However, with the mixture of organisms present in hay, the dilution stages proved completely unreliable; in one test a tenfold dilution series decreased numbers by only one fourth at each stage (compare Brierley, Jewson \& Brierley, 1927). Dilution methods were therefore given up in favour of the Andersen sampler (Andersen, 1958). In this instrument the air enters a circular orifice and is drawn in succession through a series of six circular plates, each perforated with $\mathbf{4 0 0}$ holes through which spores are impacted directly on the surface of sterile medium in Petri dishes. Succeeding stages in the series have

Table 1. Numbers of spores blown from successive samples taken from single bales of hay

\begin{tabular}{|c|c|c|}
\hline \multicolumn{3}{|c|}{ Good hay (L) } \\
\hline & Moulds & $\begin{array}{l}\text { Actinomycetes } \\
\text { (and bacteria) }\end{array}$ \\
\hline & \multicolumn{2}{|c|}{$\begin{array}{c}\text { Spores } \\
\text { (millions/g. dry wt. hay) }\end{array}$} \\
\hline Date & $\longrightarrow$ & 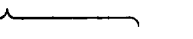 \\
\hline 17. vi. 59 & 0.28 & $1 \cdot 69$ \\
\hline 19. vi. 59 & 0.07 & 0.58 \\
\hline 30. vi. 59 & $0 \cdot 27$ & 0.07 \\
\hline 7. vii. 59 & $0 \cdot 19$ & 0.24 \\
\hline 6. x. 59 & 0.03 & 0.24 \\
\hline
\end{tabular}

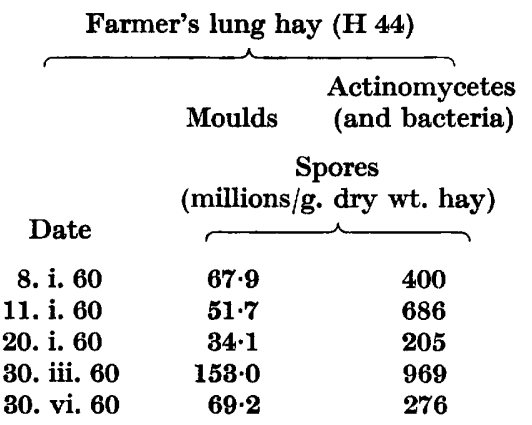

progressively smaller holes; the largest particles are deposited in the first dish and the smallest in the last. The Andersen sampler was placed horizontally on the axis of the wind-tunnel with the orifice facing the wind (Fig. 1), and was operated at $25 \mathrm{l}$. air/min. According to Andersen, the first two stages with larger holes retain the 'non-penetrating' fraction, while stages 3 to 6 retain the 'penetrating' fraction, as defined above. Flow into the sampler is non-isokinetic and, at the wind speed of $4.2 \mathrm{~m}$. $/ \mathrm{sec}$., the speed of air-flow through the orifice is only $16 \%$ of the ambient tunnel wind speed; the largest particles in the non-penetrating range therefore are over-collected. On the other hand some of the large particles are lost by impaction on the front of the first perforated plate.

In an actual test of a sample of hay the cascade impactor was used during the first $3 \mathrm{~min}$. of shaking, and then several sets of Petri dishes were exposed in the Andersen sampler during the fourth minute. After exposure, the sets of malt extract agar (2.0\% malt extract, $2 \cdot 0 \%$ agar, about $\mathrm{pH} 5 \cdot 7)$, with 20 units penicillin/ $\mathrm{ml}$. and 40 units streptomycin $/ \mathrm{ml}$. to suppress bacteria, were incubated at $25^{\circ}$ and $40^{\circ}$. Plates of half-strength Oxoid nutrient agar $(\mathrm{pH} \mathrm{7.4})$ with $0.5 \mathrm{mg}$. actidione $/ \mathrm{ml}$. to suppress moulds were incubated at $25^{\circ}, 40^{\circ}$ and $60^{\circ}$. All plates were poured the day previously to allow the surface to dry. Each set of six plates was exposed in the Andersen sampler for 15 sec., giving 5 l. of air through the apparatus (occasionally to avoid overcrowding only 5 sec. were given). Although exposures were made during the fourth minute of shaking after the cascade impactor runs, when most of the spores were blown off, the plates were frequently overloaded. This was shown by the presence of the maximum possible number of 400 colonies/plate, many of them multiple infections for which no allowance has been made in Tables 3 and 5 to 7; counts from the Andersen sampler are therefore underestimates. 


\section{RESULTS}

\section{Examination of farm hays}

Results of examining different batches of hay are presented in four groups: $a$ good hays from a hay-drying experiment; $b$ other good hays; $c$ mouldy hays and hays associated with animal diseases; $d$ farmer's lung hays. Classification into groups $b$ and $c$ was made on the results of the examination, but classification into group $d$ was based on the diagnosis of farmer's lung in the patient by the physician. Table 2 shows the presence and abundance (over 2 million/g. hay) of spores in the different groups of hay as seen on the cascade impactor slides. Table 3 shows the presence and abundance (over twenty-five colonies isolated) of organisms isolated from the four groups of hay with the Andersen sampler.

Table 2. Presence of spores counted on the cascade impactor slides

(Brackets indicate number of samples containing over 2 million spores/g. dry wt. hay.)

\begin{tabular}{|c|c|c|c|c|}
\hline & $\begin{array}{c}\text { Experimental } \\
\text { hays, } \\
\text { group } a\end{array}$ & $\begin{array}{l}\text { Good } \\
\text { hays, } \\
\text { group } b\end{array}$ & $\begin{array}{l}\text { Mouldy } \\
\text { hays, } \\
\text { group c }\end{array}$ & $\begin{array}{c}\text { Farmer's } \\
\text { lung hays, } \\
\text { group } d\end{array}$ \\
\hline & \multicolumn{4}{|c|}{ No. batches tested } \\
\hline & 10 & 18 & 17 & 14 \\
\hline Actinomycetes & $10(3)$ & $18(5)$ & $17(15)$ & $14(14)$ \\
\hline Acremoniella atra & $\mathbf{0}$ & 0 & 1 & 2 \\
\hline Alternaria spp. & 1 & 1 & 1 & $\mathbf{0}$ \\
\hline Aspergillus and Penicillium spp. & $10(7)$ & $16(5)$ & $17(7)$ & $14(7)$ \\
\hline Chaetomium spp. & 0 & 0 & 0 & 5 \\
\hline Cladosporium spp. & $\mathbf{5}$ & 2 & 10 & 7 \\
\hline Epicoccum spp. & 1 & $\mathbf{0}$ & 4 & $\mathbf{1}$ \\
\hline Humicola lanuginosa & $\mathbf{0}$ & $\mathbf{0}$ & 9 & $13(3)$ \\
\hline H. stellata & $\mathbf{0}$ & $\mathbf{0}$ & 2 & $\mathbf{3}$ \\
\hline Mucoraceae & 2 & $7(1)$ & $13(3)$ & $11(3)$ \\
\hline Trichothecium roseum & $\mathbf{0}$ & 2 & 3 & 5 \\
\hline Number of categories & $6(2)$ & $6(8)$ & $10(3)$ & $10(4)$ \\
\hline
\end{tabular}

Group a: good hays from a hay-drying experiment (Table 4). In July 1958 batches of hay were dried experimentally at the National Institute of Agricultural Engineering, Silsoe, Bedfordshire. Samples were obtained and tested in February and March 1959. Spores of Aspergillus glaucus and Penicillium type were the ones present in large quantities, and occurred in the swath dried hays $(\mathrm{H} 7,10,11)$. A few spores of Cladosporium, Alternaria, Epicoccum and of Mucoraceae were also present. These hays never had more than 3 million actinomycete spores/g. hay. These batches of good hay were studied with alginate wool filters before the Andersen sampler was used. The fungi isolated were mainly mesophilic, the most numerous were $\boldsymbol{A}$. glaucus (especially in $\mathrm{H}$ 10) and Penicillium spp. Absidia sp. was isolated from $\mathrm{H} 6$ and $7, A$. fumigatus from only $\mathrm{H} 8$ and 13, and Hemispora stellata from H 5, 10, 11, and 13. Neither Mucor pusillus nor Humicola lanuginosa were isolated.

Group b: other good hays (Table 5). Samples classed as 'good hay' had very different microbial contents, ranging from $\mathrm{H} \mathrm{33}$, of which the farmer who sent it was justly proud, to others yielding several million spores/g. hay. The $\mathrm{pH}$ values varied from 4.5 to 6.5 (av. of 13 hays, $\mathrm{pH} \mathrm{5.9}$ ). The range of microbial content was 
generally similar to the experimental hays of Table 4. Some samples, however, contained more actinomycete spores than moulds. Aspergillus glaucus was the most plentiful mould spore, especially in $\mathrm{H} \mathrm{56,58,68}$ and 69. Hemispora stellata was

Table 3. Isolation of organisms in culture

(Brackets indicate number of samples with over 25 isolations.)

\begin{tabular}{|c|c|c|c|c|}
\hline & $\begin{array}{c}\text { Experimental } \\
\text { hays, } \\
\text { group } a\end{array}$ & $\begin{array}{l}\text { Good } \\
\text { hays, } \\
\text { group b }\end{array}$ & $\begin{array}{l}\text { Mouldy } \\
\text { hays, } \\
\text { group } c\end{array}$ & $\begin{array}{c}\text { Farmer's } \\
\text { lung hays, } \\
\text { group } d\end{array}$ \\
\hline & & No. batch & tested & \\
\hline & 10 & 18 & 17 & 14 \\
\hline Fungi & & & & \\
\hline Absidia (mainly ramosa) & 2 & 15 & $13(4)$ & $13(2)$ \\
\hline A. corymbifera & $\mathbf{0}$ & 2 & $\mathbf{0}$ & 0 \\
\hline Acremoniella atra & 1 & $\mathbf{0}$ & $\mathbf{0}$ & $\mathbf{0}$ \\
\hline Alternaria spp. & 1 & $\mathbf{0}$ & $\mathbf{3}$ & $\mathbf{0}$ \\
\hline Aspergillus spp. (total) & $8(1)$ & $17(6)$ & $17(10)$ & 14. (10) \\
\hline A. fumigatus & 2 & 11 & $15(5)$ & $13(5)$ \\
\hline A. glaucus & $7(1)$ & $15(6)$ & $12(7)$ & $12(7)$ \\
\hline A. nidulans & 1 & 13 & $11(4)$ & $8(1)$ \\
\hline A. niger & $\mathbf{0}$ & 0 & 5 & 0 \\
\hline A. ochraceus & 0 & $\mathbf{0}$ & 1 & $\mathbf{0}$ \\
\hline A. terreus & $\mathbf{0}$ & 2 & 6 & $4(1)$ \\
\hline A. versicolor & $\mathbf{3}$ & $\mathbf{1}$ & $7(2)$ & $7(1)$ \\
\hline Botrytis spp. & $\mathbf{5}$ & 1 & 3 & 3 \\
\hline Candida spp. & 0 & 8 & 3 & $\mathbf{3}$ \\
\hline Cephalosporium sp. & $\mathbf{1}$ & $\mathbf{0}$ & 1 & $\mathbf{0}$ \\
\hline Chaetomium sp. & 1 & $\mathbf{3}$ & $\mathbf{5}$ & 4 \\
\hline Cladosporium sp. & $\mathbf{2}$ & $15(2)$ & $13(4)$ & $13(1)$ \\
\hline Epicoccum sp. & $\mathbf{2}$ & 1 & 1 & 0 \\
\hline Fusarium sp. & $\mathbf{0}$ & $\mathbf{0}$ & 1 & $\mathbf{0}$ \\
\hline Geomyces type & $\mathbf{3}$ & 2 & 0 & 1 \\
\hline Hemispora stellata & 4 & 3 (3) & $3(1)$ & 0 \\
\hline Humicola lanuginosa & $\mathbf{0}$ & 6 & 4 & $9(3)$ \\
\hline H. stellata & $\mathbf{0}$ & $\mathbf{0}$ & 0 & 1 \\
\hline Mucor pusillus & $\mathbf{0}$ & 9 & $12(3)$ & $10(1)$ \\
\hline M. spinus & $\mathbf{0}$ & $\mathbf{0}$ & 0 & 1 \\
\hline Paecilomyces sp. & $\mathbf{0}$ & 6 & 3 (2) & 9 \\
\hline Papularia sp. & $\mathbf{0}$ & 1 & 1 & 0 \\
\hline Penicillium spp. & 8 & 16 & $13(4)$ & $10(1)$ \\
\hline Pullularia sp. & $\mathbf{0}$ & 1 & 1 & 1 \\
\hline Rhizopus sp. & $\mathbf{0}$ & 1 & 1 & 1 \\
\hline Scopulariopsis brevicaulis & $\mathbf{3}$ & 1 & 3 & $4(2)$ \\
\hline Sporendonema type & $\mathbf{0}$ & $\mathbf{0}$ & $\mathbf{0}$ & 1 \\
\hline Sporotrichum sp. & 1 & $\mathbf{0}$ & $\mathbf{0}$ & $\mathbf{3}$ \\
\hline Stachybotrys sp. & $\mathbf{0}$ & $\mathbf{0}$ & $\mathbf{0}$ & 1 \\
\hline Stysanus spp. & $\mathbf{1}$ & $\mathbf{0}$ & 1 & $\mathbf{0}$ \\
\hline Trichoderma viride & $\mathbf{0}$ & $\mathbf{0}$ & $\mathbf{0}$ & $\mathbf{2}$ \\
\hline Trichothecium roseum & $\mathbf{0}$ & 1 & $3(1)$ & 0 \\
\hline Yeasts & 1 & 7 & 5 & $3(1)$ \\
\hline Sterile mycelium or unidentified & - & 10 & 7 & 5 \\
\hline Number of categories & $19(1)$ & $25(3)$ & $29(11)$ & $26(12)$ \\
\hline Bacteria & & & & \\
\hline $\mathbf{2 4}^{\circ}$ orange & - & 3 & 6 (4) & $10(\mathbf{3})$ \\
\hline others & $\mathbf{1}$ & 16 (5) & 16 (3) & $13(4)$ \\
\hline $40^{\circ}$ Bacillus cereus-mycoides & - & 11 & 16 (1) & 13 (3) \\
\hline others & - & 13 & $17(3)$ & $11(3)$ \\
\hline Number of categories & 1 & $4(1)$ & $4(4)$ & $4(4)$ \\
\hline
\end{tabular}


Table 3 (cont.)

\begin{tabular}{|c|c|c|c|c|c|}
\hline & & $\begin{array}{l}\text { Experimental } \\
\text { hays, } \\
\text { group } a\end{array}$ & $\begin{array}{l}\text { Good } \\
\text { hays, } \\
\text { group } b\end{array}$ & $\begin{array}{l}\text { Mouldy } \\
\text { hays, } \\
\text { group } c\end{array}$ & $\begin{array}{l}\text { Farmer's } \\
\text { lung hays, } \\
\text { group } d\end{array}$ \\
\hline Actino & mycetes & & & & \\
\hline $24^{\circ}$ & & 2 & 15 & $17(8)$ & $13(8)$ \\
\hline $40^{\circ}$ & whitish & - & $15(12)$ & $17(10)$ & $15(9)$ \\
\hline & blue & - & 2 & $8(2)$ & $11(4)$ \\
\hline & grey & - & $10(1)$ & 12 & $12(3)$ \\
\hline & thin & - & 3 & $3(1)$ & 0 \\
\hline & small & - & $4(2)$ & $11(6)$ & $13(12)$ \\
\hline $60^{\circ}$ & white & - & $11(3)$ & $13(7)$ & $13(11)$ \\
\hline & small & - & 0 & $4(3)$ & $9(9)$ \\
\hline Numb & of categories & 1 & $7(4)$ & $8(7)$ & $7(7)$ \\
\hline Total & lumber of categories & $21(1)$ & $36(8)$ & $41(22)$ & $37(23)$ \\
\hline
\end{tabular}

Table 4. Microbial content of good hay (group a) artificially dried at N.I.A.E., Silsoe, Bedfordshire, in July 1958

\begin{tabular}{|c|c|c|c|}
\hline & & Visual e & mination \\
\hline & & Moulds & $\begin{array}{l}\text { Actino- } \\
\text { mycetes }\end{array}$ \\
\hline & & $\underset{\text { (millions/g. }}{\mathbf{S}_{\mathbf{r}} / \mathrm{g}}$ & es \\
\hline Hay ref. no. & Treatment & & \\
\hline H 5 (D. 1) & Tunnel dried & $4 \cdot 5$ & $1 \cdot 1$ \\
\hline H 6 (D. 2) & Hut rack dried & $\mathbf{2 \cdot 9}$ & $0 \cdot 8$ \\
\hline H 7 (D. 3) & Swath dried & $\mathbf{3 2} \cdot \mathbf{3}$ & $2 \cdot 4$ \\
\hline H 8 (C. 1) & Tunnel dried & $\mathbf{0} \cdot \mathbf{1}$ & $0 \cdot 6$ \\
\hline H 9 (C. 2) & Hut rack dried & $\mathbf{2 \cdot 1}$ & $\mathbf{0 . 9}$ \\
\hline H 10 (C. 3) & Swath dried & $\mathbf{9 \cdot 2}$ & $1 \cdot 3$ \\
\hline H 11 (C. 4) & Swath dried & $10 \cdot 3$ & $2 \cdot 5$ \\
\hline H 12 (E. 1) & Barn dried & $5 \cdot 6$ & $1 \cdot 6$ \\
\hline H 13 (E. 2) & Hut rack dried & $2 \cdot 8$ & 0.5 \\
\hline H 14 (E. 3) & Swath dried & $6 \cdot 9$ & $\mathbf{3 \cdot 0}$ \\
\hline
\end{tabular}

seen in $\mathrm{H} 37$ and 69, and in $\mathrm{H} 56 \quad 0.68$ million spores/g. hay were counted. Mucoraceae spores were seen in only seven of these hays (Table 2) and only in $\mathbf{H} \mathbf{1 7}$ reached 2 million/g. The hays from groups $a$ and $b$ contain fungi, such as Cladosporium, Epicoccum, Helminthosporium and Alternaria, which are the normal constituents of fine weather air-spora; but hays of poorer quality had moulded with $\boldsymbol{A}$. glaucus, $\boldsymbol{H}$. stellata and Penicillium spp. In culture, the only abundant moulds

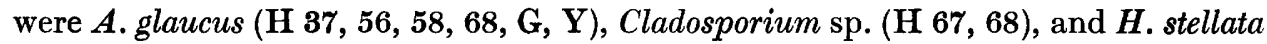
(H 37, 56 and 69 with 14,000 viable spores/g.). A few $A$. fumigatus were isolated from all except $\mathbf{H} 33,37, \mathrm{~B}, \mathrm{G}$, and N, of $A$ bsidia spp. from all except $\mathbf{H} 19,33$, and 71, and of Mucor pusillus from half. Very few Humicola lanuginosa were isolated from $\mathrm{H} \mathrm{69,71,} \mathrm{B,} \mathrm{G,} \mathrm{L} \mathrm{and} \mathrm{Y}$. Bacteria and actinomycetes were isolated from most hays but even when shown 'abundant' (Table 6) were many fewer than in hays of groups $c$ and $d$.

Group c: mouldy hays (Table 6). These included batches associated with diseases of farm animals and various other samples sent to us as mouldy, but excluding any 
samples sent as implicated in producing farmer's lung disease; the latter are dealt with in group $d$. The $\mathrm{pH}$ value ranged from 4.0 to $8 \cdot 3$; the average of 13 hays ( $\mathrm{pH} \mathrm{6.5)}$ was very similar to that of the good hays of group $b$. The hays of group $c$ had very different microbial contents, and spores of moulds or actinomycetes (or both) usually numbered between 10 and 100 million/g. Aspergillus and Penicillium type spores were numerous in $\mathrm{H} \mathrm{27,49,59,63,66,74}$, and 79, Mucoraceae were abundant in only $\mathrm{H} 27$ and 76. Humicola lanuginosa was present in $\mathrm{H} 1,27,40,41,49,59$, 61,63 and $66, H$. stellata in $\mathrm{H} 27$ and 41 .

Table 5. Microbial content of good hay samples, group b

\begin{tabular}{|c|c|c|c|c|c|c|c|}
\hline \multirow{3}{*}{$\begin{array}{l}\text { Hay } \\
\text { ref. } \\
\text { no. } \\
\text { H } 17\end{array}$} & \multirow{2}{*}{ Origin } & \multirow{3}{*}{$\begin{array}{c}\text { pH } \\
\text { value } \\
-\end{array}$} & \multirow{2}{*}{\multicolumn{2}{|c|}{$\overbrace{\begin{array}{c}\text { Actino- } \\
\text { Spores } \\
\text { Mycetes }\end{array}}^{\text {Visual examination }}$}} & \multicolumn{3}{|c|}{ From culture } \\
\hline & & & & & $\begin{array}{l}\text { Moulds } \\
\text { (thousa }\end{array}$ & $\begin{array}{l}\text { Actino- } \\
\text { mycetes } \\
\text { Spores } \\
\text { ads/g. dry }\end{array}$ & $\begin{array}{l}\text { Bacteria } \\
\text { wt. hay) }\end{array}$ \\
\hline & $\begin{array}{l}\text { Weybridge, Surrey, as fed to cows at } \\
\text { Central Veterinary Laboratory }\end{array}$ & & $\mathbf{2 . 7 5}$ & $1 \cdot 34$ & 一 & - & - \\
\hline H 19 & $\begin{array}{l}\text { Plumpton, Sussex (normal hay fed to cows } \\
\text { on a farm where mycotic abortion had } \\
\text { occurred) }\end{array}$ & - & 0.77 & $\mathbf{1 \cdot 6 8}$ & - & - & 一 \\
\hline H 33 & Leckhampstead, Newbury, Berks. & - & $\mathbf{0} \cdot \mathbf{1 7}$ & 0.22 & - & - & 一 \\
\hline H 37 & $\begin{array}{l}\text { Dulverton, Som. (1959 hay 'inactive'; } \\
\text { cf. H } 36 \text { (Table 7)) }\end{array}$ & $6 \cdot 4$ & $\mathbf{2 \cdot 6 0}$ & $2 \cdot 90$ & $26 \cdot 6$ & $\mathbf{4} \cdot \mathbf{8 0}$ & $\mathbf{0 . 4 1}$ \\
\hline H 38 & $\begin{array}{l}\text { Weymouth, Dorset (associated with case } \\
\text { of sarcoid) }\end{array}$ & $6 \cdot 5$ & $\mathbf{8 \cdot 2}$ & $\mathbf{2 \cdot 4}$ & - & - & - \\
\hline H 56 & $\begin{array}{l}\text { Lympsham, nr. Weston-super-Mare, } \\
\text { Somerset (associated with non-seasonal } \\
\text { allergic hay fever and asthma) }\end{array}$ & $6 \cdot 2$ & $\mathbf{8 \cdot 5}$ & $\mathbf{1 \cdot 0}$ & $17 \cdot 6$ & $5 \cdot 86$ & 3.08 \\
\hline H 58 & Banwell, Somerset & $6 \cdot 3$ & $\mathbf{5 \cdot 4 4}$ & $8 \cdot 17$ & $7 \cdot 39$ & $7 \cdot 87$ & $0 \cdot 76$ \\
\hline H 67 & Beacon, Honiton, Devon, old ley, 1961 & $6 \cdot 3$ & $\mathbf{0 . 2 1}$ & $0 \cdot 67$ & $1 \cdot 73$ & $0 \cdot 19$ & $\mathbf{1 \cdot 1 2}$ \\
\hline H 68 & Luppitt, Honiton, Devon, 2nd year ley, 1961 & $6 \cdot 2$ & $6 \cdot 89$ & 0.25 & $17 \cdot 9$ & 0.80 & $0 \cdot 28$ \\
\hline H 69 & Luppitt, Honiton, Devon, 3rd year ley, 1961 & $5 \cdot 4$ & $6 \cdot 23$ & $0 \cdot 19$ & $\mathbf{9 \cdot 8 6}$ & $0 \cdot 75$ & $0 \cdot 66$ \\
\hline H 71 & Knighton, Radnorshire, 2nd year ley, 1961 & $6 \cdot 2$ & $\mathbf{0 . 2 3}$ & 1.89 & $1 \cdot 18$ & 0.72 & $0 \cdot 85$ \\
\hline H 72 & Knighton, Radnorshire, meadow hay, 1961 & $6 \cdot 2$ & 0.81 & 0.72 & $1 \cdot 99$ & $1 \cdot 06$ & $\mathbf{2 \cdot 8 4}$ \\
\hline $\mathbf{H} 7 \mathbf{8}$ & Knighton, Radnorshire, 1st year ley, 1961 & $4 \cdot 5$ & 0.11 & 0.41 & 0.97 & $0 \cdot 86$ & $\mathbf{1 \cdot 2 5}$ \\
\hline $\mathbf{B}$ & Rothamsted, Harpenden, Herts., 1961 & $6 \cdot 0$ & 0 & 0.08 & $\mathbf{0 . 3 0}$ & $\mathbf{0} \cdot \mathbf{3 7}$ & 1.58 \\
\hline $\mathbf{G}$ & Rothamsted, Harpenden, Herts., 1961 & $6 \cdot 3$ & 0.76 & 0.07 & $18 \cdot 9$ & 0.94, & $\mathbf{0 \cdot 8 2}$ \\
\hline $\mathbf{L}$ & Rothamsted, Harpenden, Herts., 1959 & - & $0 \cdot 41$ & $\mathbf{0 . 5 4}$ & - & - & - \\
\hline $\mathbf{N}$ & Rothamsted, Harpenden, Herts., 1959 & - & $\mathbf{2 \cdot 2 1}$ & $5 \cdot 6$ & 一 & - & - \\
\hline $\mathbf{Y}$ & Rothamsted, Harpenden, Herts., 1960 & $\mathbf{5 \cdot 2}$ & $1 \cdot 43$ & $2 \cdot 16$ & $4 \cdot 40$ & $4 \cdot 78$ & $0 \cdot 73$ \\
\hline
\end{tabular}

In culture, many thermophilic moulds were abundant: Absidia spp. (H 61, 75, 76, 79), Aspergillus fumigatus (H 1, 63, 66, 76, 79), A. nidulans $(\mathrm{H} \mathrm{1,} \mathrm{66,} \mathrm{76,} \mathrm{79),}$ Mucor pusillus (H 59, 66, 79) and Paecilomyces sp. (H 59, 75). Abundant mesophilic moulds were: $A$. glaucus (H 1, 46, 49, 53, 61, 66, 74), A. versicolor (H 59, 74), Cladosporium sp. (H 49, 53, 59, 74), Hemispora stellata (H 74), Penicillium spp. $(\mathbf{H ~} 1,74,76,79)$ and Trichothecium roseum (H 79). Humicola lanuginosa was isolated from $\mathrm{H} 1,40,53$ and 63. Bacteria and actinomycetes were isolated from all samples. Bacteria isolated at $24^{\circ}$ were abundant in $H 1,16,59,61,66$ and 74 , and at $40^{\circ}$ in $\mathrm{H} \mathrm{1}, 16,59$ and 66. Actinomycetes isolated at $24^{\circ}$ were abundant in $\mathrm{H} 1$, $16,27,59,61,63,76$ and 79 ; at $40^{\circ}$ in $\mathrm{H} \mathrm{1}, 16,27,41,59,61,63,75,76$ and 79 ; and at $60^{\circ}$ in $\mathrm{H} 1,16,59,61,63,75$ and 79 . 
Group d: farmer's lung hays (Table 7). Fourteen samples of hay associated with cases of farmer's lung were examined between 1958 and 1961. Some of these were browner than normal hay, but they differed from the typical brown hay described by Truninger (1929) which was usually acid (pH 4.4-5.1). Characteristically farmer's lung hay is less acid than the 'good' or 'mouldy' hays of groups $b$ and $c$, between $\mathrm{pH} 6 \cdot 0$ and $7 \cdot 5$ (average of 12 hays, $\mathrm{pH} 7 \cdot 1$ ). The outstanding character of group $d$ hay was the large content of actinomycete spores, over 60 million/g. (except $\mathbf{H} 45$ with only 5 million). Fungus spores are often abundant. Aspergillus

Table 6. Microbial content of mouldy hays and hays associated with animal diseases, group $\mathrm{c}$

\begin{tabular}{|c|c|c|c|c|c|c|c|}
\hline \multirow[b]{2}{*}{$\begin{array}{l}\text { Hay } \\
\text { ref. } \\
\text { no. }\end{array}$} & \multirow[b]{2}{*}{ Origin } & \multicolumn{3}{|c|}{ Visual examination } & \multicolumn{3}{|c|}{ From culture } \\
\hline & & $\underset{\text { value }}{\mathbf{p H}}$ & \multicolumn{2}{|c|}{$\begin{array}{c}\text { Moulds mycetes } \\
\text { Spores } \\
\text { (millions/g. } \\
\text { dry wt. hay) }\end{array}$} & \multirow[t]{2}{*}{$\begin{array}{l}\text { Moulds } \\
\text { (thousa }\end{array}$} & $\begin{array}{r}\text { Actino- } \\
\text { mycetes } \\
\text { Spores } \\
\text { nds/g. dry }\end{array}$ & $\begin{array}{l}\text { Bacteris } \\
\text { wt. hay) }\end{array}$ \\
\hline \multicolumn{7}{|c|}{ Relatively good hays associated with animal diseases } & \\
\hline H 46 & $\begin{array}{l}\text { Haslemere, Surrey (mycotic abortion in } \\
\text { cows) }\end{array}$ & $6 \cdot 1$ & $1 \cdot 1$ & 4.0 & $\mathbf{3 \cdot 4 4}$ & $2 \cdot 64$ & $1 \cdot 12$ \\
\hline H 49 & $\begin{array}{l}\text { Norwich, Norfolk (mucormycotic abomasal } \\
\text { ulcers in Jersey calves) }\end{array}$ & 6.2 & $1 \cdot 1$ & $4 \cdot 6$ & $9 \cdot 49$ & $1 \cdot 23$ & $1 \cdot 52$ \\
\hline H 53 & Reading, Berks. (buccal ulceration in cows) & $6 \cdot 0$ & $\mathbf{1} \cdot \mathbf{0}$ & $4 \cdot 0$ & $\mathbf{2 1 \cdot 4}$ & 3.92 & 0.5 \\
\hline H 75 & $\begin{array}{l}\text { Horsham, Sussex, } 1960 \text { hay (mycotic abor- } \\
\text { tion in cows) }\end{array}$ & $4 \cdot 0$ & $0 \cdot 8$ & $\mathbf{1 \cdot 3 6}$ & $\mathbf{3 \cdot 2 5}$ & $4 \cdot 18$ & $0 \cdot 4$ \\
\hline \multicolumn{8}{|c|}{ Mouldy hays associated with animal diseases } \\
\hline H 1 & Reading, Berks. (acute pneumonia of calves) & - & $71 \cdot 5$ & $\mathbf{8 0 \cdot 2}$ & $10 \cdot 83$ & $\mathbf{5 6} \cdot \mathbf{3}$ & $\mathbf{0 . 9 2}$ \\
\hline H 16 & Cambridge (aspergillosis of lambs) & - & $\mathbf{0 . 7 6}$ & $190 \cdot 0$ & 4.32 & $79 \cdot 8$ & $\mathbf{2 5 \cdot 2}$ \\
\hline H 74 & $\begin{array}{l}\text { Horsham, Sussex, } 1961 \text { hay (mycotic } \\
\text { abortion in cows) }\end{array}$ & $\mathbf{5} \cdot \mathbf{8}$ & $15 \cdot 1$ & $\mathbf{2 \cdot 1 6}$ & $4 \cdot 45$ & $\mathbf{0 \cdot 3 4}$ & $\mathbf{0 \cdot 9 8}$ \\
\hline H 76 & $\begin{array}{l}\text { Horsham, Sussex, } 1960 \text { hay (mycotic } \\
\text { abortion in cows) }\end{array}$ & $\mathbf{5 \cdot 3}$ & $42 \cdot 0$ & $8 \cdot 47$ & $48 \cdot 2$ & $58 \cdot 3$ & $0 \cdot 63$ \\
\hline H 79 & $\begin{array}{l}\text { Basingstoke, Hants. (coughing in Guernsey } \\
\text { cows) }\end{array}$ & $5 \cdot 7$ & $19 \cdot 5$ & $1 \cdot 34$ & $57 \cdot 9$ & 0.54 & $\mathbf{0 \cdot 2 1}$ \\
\hline \multicolumn{8}{|c|}{ Other mouldy hays } \\
\hline H 27 & Liskeard, Cornwall & - & $100 \cdot 0$ & $49 \cdot 6$ & - & - & - \\
\hline $\mathbf{H} 40$ & Brampton, Cumb., 1958 ley hay & $4 \cdot 5$ & $4 \cdot 2$ & $251 \cdot 0$ & - & - & - \\
\hline H 41 & $\begin{array}{l}\text { Brampton, Cumb., } 1958 \text { old land meadow } \\
\text { hay }\end{array}$ & $8 \cdot 3$ & $4 \cdot 1$ & $198 \cdot 0$ & - & - & - \\
\hline H 42 & Brampton, Cumb., 1958 meadow hay & $7 \cdot 4$ & $\mathbf{2 \cdot 2}$ & $205 \cdot 0$ & - & - & - \\
\hline H 59 & Banwell, Somerset, old pasture hay & $6 \cdot 8$ & $7 \cdot 4$ & $41 \cdot 9$ & $19 \cdot 5$ & $\mathbf{3 1} \cdot \mathbf{3}$ & $\mathbf{2 2 \cdot 7}$ \\
\hline H 61 & Berkeley, Glos. & $7 \cdot 5$ & $2 \cdot 6$ & $28 \cdot 0$ & 11.2 & $60 \cdot 9$ & $13 \cdot 0$ \\
\hline H 63 & $\begin{array}{l}\text { Aberystwyth (inactive with patient of } \\
\text { H 64; Table 7) }\end{array}$ & $6 \cdot 1$ & $4 \cdot 5$ & $\mathbf{2 5} \cdot 6$ & $4 \cdot 55$ & $\mathbf{3 9} \cdot 8$ & $\mathbf{1} \cdot \mathbf{0 3}$ \\
\hline H 66 & Rothamsted, Harpenden, Herts., 1961 hay & - & $27 \cdot 8$ & $\mathbf{3 \cdot 9 5}$ & 4.56 & 0.24 & $\mathbf{2 3 \cdot 2}$ \\
\hline
\end{tabular}

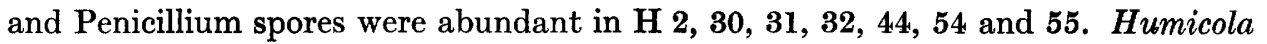
lanuginosa was abundant in $\mathrm{H} \mathrm{18,43}$ and 44, and the Mucoraceae in $\mathrm{H} \mathrm{30,31}$ and 44.

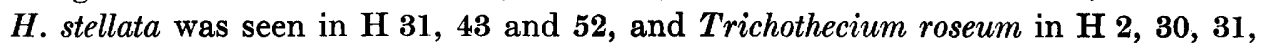
44 and 64.

In culture, many thermophilic moulds were abundant: Absidia spp. (H 30, 31), Aspergillus fumigatus (H 30, 44, 52, 54, 64), A. nidulans (H 30), A. terreus (H 30), Humicola lanuginosa (H 18, 43, 64) and Mucor pusillus (H 44). Mesophilic moulds 
that were abundant were: $A$. glaucus $(\mathrm{H} \mathrm{2,} \mathrm{31,} \mathrm{32,} \mathrm{36,} \mathrm{44,} \mathrm{45,} \mathrm{65),} \mathrm{A.} \mathrm{versicolor}(\mathrm{H} \mathrm{2),}$ Cladosporium sp. (H 30), Penicillium spp. (H 30), Scopulariopsis brevicaulis (H 2, 31) and a yeast ( $\mathrm{H} \mathrm{32}$ ). Humicola stellata was isolated from $\mathrm{H} 54$ and a thermophilic

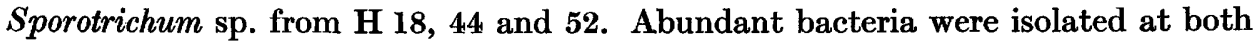
$24^{\circ}$ and $40^{\circ}$ from $\mathrm{H} \mathrm{30}, 31,32,44$ and 64. Actinomycetes isolated at $24^{\circ}$ were

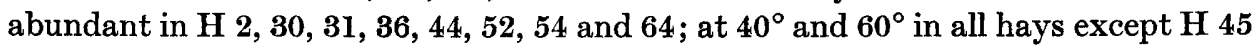
(and $\mathrm{H} 52$ which was not tested at $60^{\circ}$ ). Although $\mathrm{H} 45$ seems out of place in this group, the diagnosis of farmer's lung was well established and the discrepancy may

Table 7. Microbial content of farmer's lung hays, group $\mathrm{d}$

\begin{tabular}{|c|c|c|c|c|c|c|c|c|}
\hline \multirow[b]{2}{*}{$\begin{array}{l}\text { Hay } \\
\text { ref. } \\
\text { no. }\end{array}$} & \multirow{2}{*}{ Origin } & \multirow{2}{*}{$\begin{array}{l}\mathrm{pH} \\
\text { value }\end{array}$} & \multicolumn{3}{|c|}{ Visual examination } & \multicolumn{3}{|c|}{ From culture } \\
\hline & & & $\begin{array}{l}\text { Moulds } \\
\text { (milli }\end{array}$ & $\begin{array}{l}\text { Actino- } \\
\text { mycetes } \\
\text { Spores } \\
\text { ns/g. dry }\end{array}$ & $\begin{array}{l}\text { H. lanu- } \\
\text { ginosa } \\
\text { t. hay) }\end{array}$ & $\begin{array}{l}\text { Moulds } \\
\text { (thousa }\end{array}$ & $\begin{array}{l}\text { Actino- } \\
\text { mycetes } \\
\text { Spores } \\
\text { ads/g. dry }\end{array}$ & $\begin{array}{l}\text { Bacteria } \\
\text { vt. hay) }\end{array}$ \\
\hline H 2 & $\begin{array}{l}\text { Witney, Oxfordshire (acute } \\
\text { thresher's lung) }\end{array}$ & $7 \cdot 5$ & $11 \cdot 0$ & $134 \cdot 0$ & 0.08 & $11 \cdot 3$ & $70 \cdot 3$ & $1 \cdot 01$ \\
\hline H 18 & Near Aberystwyth & $7 \cdot 0$ & $\mathbf{3} \cdot 6$ & $168 \cdot 0$ & $\mathbf{1} \cdot \mathbf{1 0}$ & $4 \cdot 84$ & $16 \cdot 2$ & $4 \cdot 72$ \\
\hline H 30 & Whitchurch, Shropshire & $6 \cdot 0$ & $\mathbf{8 6 \cdot 2}$ & $132 \cdot 0$ & $13 \cdot 8$ & $148 \cdot 2$ & $103 \cdot 3$ & $\mathbf{5 3 \cdot 8 5}$ \\
\hline H 31 & $\begin{array}{l}\text { Broadwoodkelly, nr. Winkleigh, } \\
\text { Devon (1958 hay) }\end{array}$ & $7 \cdot 6$ & $12 \cdot 0$ & $\mathbf{3 6 3 \cdot 0}$ & $0 \cdot 64$ & $6 \cdot 24$ & $60 \cdot 7$ & $12 \cdot 52$ \\
\hline H 32 & $\begin{array}{l}\text { Broadwoodkelly, nr. Winkleigh, } \\
\text { Devon (1959 hay) }\end{array}$ & $7 \cdot \mathbf{3}$ & $7 \cdot 8$ & $480 \cdot 0$ & 0.97 & $18 \cdot 7$ & $85 \cdot 7$ & $9 \cdot 45$ \\
\hline H 36 & $\begin{array}{l}\text { Dulverton, Som. (1958 hay } \\
\text { 'active') cf. H 37, Table } 5\end{array}$ & $6 \cdot 6$ & $9 \cdot 1$ & $91 \cdot 3$ & $\mathbf{0}$ & $13 \cdot 4$ & $216^{\cdot 6}$ & $5 \cdot 78$ \\
\hline H 43 & Awliscombe, nr. Exeter, Devon & $6 \cdot 6$ & $7 \cdot 4$ & 1282 & $\mathbf{2 \cdot 4}$ & $14 \cdot 4$ & $152 \cdot 1$ & $1 \cdot 48$ \\
\hline H 44 & $\begin{array}{l}\text { Inwardleigh, nr. Okehampton, } \\
\text { Devon }\end{array}$ & $7 \cdot 4$ & $102 \cdot 0$ & $827 \cdot 0$ & $11 \cdot 2$ & $101 \cdot 8$ & $208 \cdot 3$ & $21 \cdot 01$ \\
\hline H 45 & $\begin{array}{l}\text { Lampeter, mid-Cardiganshire } \\
\text { (farmer's lung of young lad) }\end{array}$ & $6 \cdot 7$ & $1 \cdot 0$ & $5 \cdot 0$ & $0 \cdot 1$ & $\mathbf{3} \cdot \mathbf{2 3}$ & 1.57 & $0 \cdot 68$ \\
\hline H 52 & Mid-Wales & - & $\mathbf{1 . 5}$ & $633 \cdot 0$ & $\mathbf{0 \cdot 2 4}$ & $10 \cdot 5$ & $\mathbf{2 8 \cdot 0}$ & $48 \cdot 67$ \\
\hline H 54 & Longnor, Staffordshire & $7 \cdot 4$ & $16 \cdot 6$ & $78 \cdot 3$ & $\mathbf{2 \cdot 8 7}$ & $4 \cdot 67$ & $24 \cdot 7$ & $\mathbf{2 \cdot 2 8}$ \\
\hline H 55 & Cardiff & - & $12 \cdot 4$ & $123 \cdot 0$ & 0 & $3 \cdot 75$ & $23 \cdot 2$ & 0.57 \\
\hline H 64 & $\begin{array}{l}\text { Talybont, nr. Aberystwyth, } \\
1960 \text { 'active' hay (cf. H 63, } \\
\text { Table 6) (farmer's lung for } \\
20 \text { years) }\end{array}$ & $7 \cdot 5$ & $\mathbf{2 \cdot 3}$ & $175 \cdot 0$ & 0.83 & $9 \cdot 14$ & $81 \cdot 5$ & $4 \cdot 10$ \\
\hline H 65 & $\begin{array}{l}\text { Matlock, nr. Chesterfield, } \\
\text { Derbyshire }\end{array}$ & $7 \cdot 2$ & $1 \cdot 4$ & $64 \cdot 0$ & 0.12 & 8.04 & $37 \cdot 2$ & $2 \cdot 20$ \\
\hline
\end{tabular}

mean either that the sample sent from the farm was taken accidentally from the wrong batch of hay, or the sample sent may have been unrepresentative of the batch because of the variability within the batch from uneven drying.

\section{Proportion of thermophilic organisms}

In comparison with good hay, the mouldy hay group $c$ and the farmer's lung group $d$ were characterized by many more thermophilic moulds and actinomycetes, as shown by incubation of Andersen sampler plates at different temperatures. These average values (Fig. 2), however, were calculated from individual hays which differed among themselves over a wide range of values, were uncorrected for multiple infection, and included some organisms which grow at more than one temperature. The mucoraceous moulds were more numerous at $40^{\circ}$ than at $24^{\circ}$, and in mouldy 
hays (group $c$ ) than in farmer's lung hays (group $d$ ). Aspergillus fumigatus was isolated in larger numbers at $40^{\circ}$ than at $24^{\circ}$, and Humicola lanuginosa only at $40^{\circ}$. The relatively few bacteria were isolated mainly at the lower temperatures. The most striking effect was the very large increase in thermophilic actinomycetes in farmer's lung hays (group $d$ ).
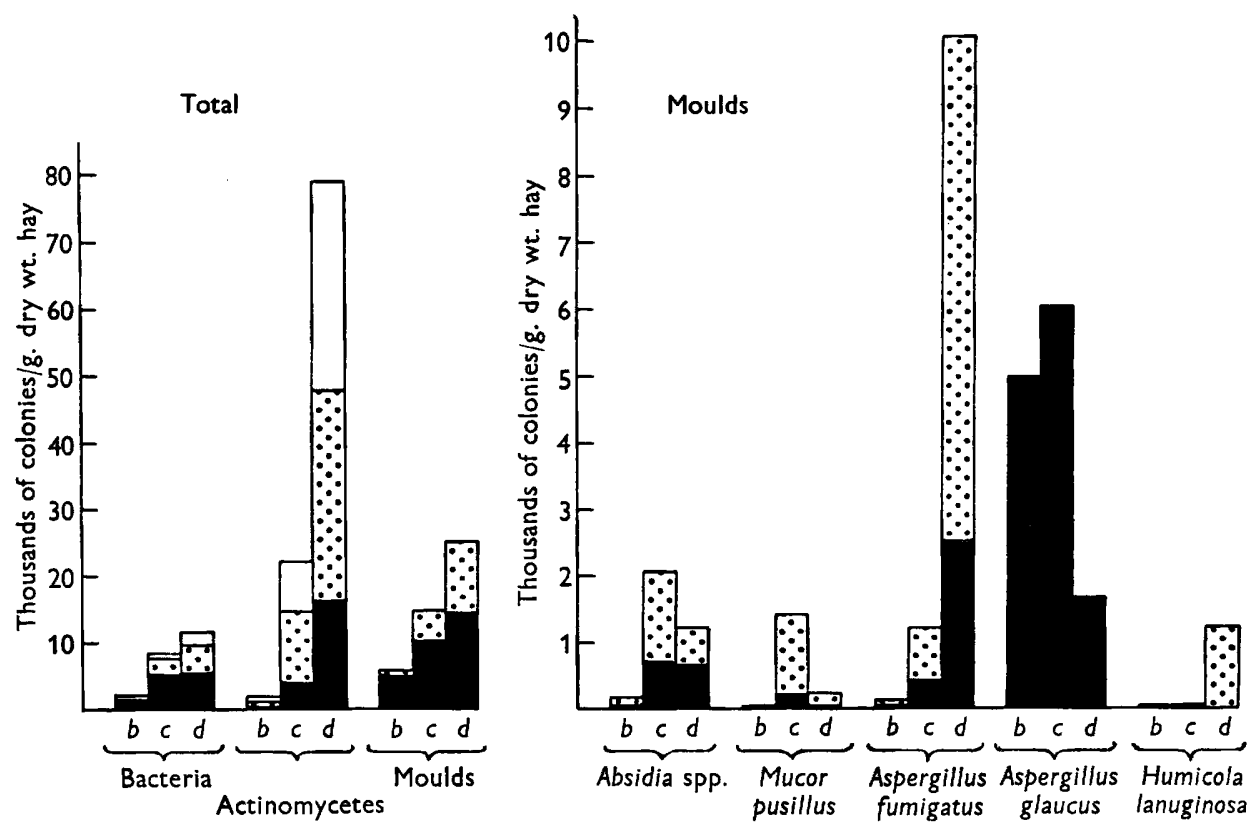

Fig. 2. Numbers of organisms isolated at $24^{\circ}$ (solid), $40^{\circ}$ (dotted) and $60^{\circ}$ (open), averages for: $(b)$ good hays; $(c)$ mouldy hays; and $(d)$ farmer's lung hays.

\section{Other components of dusts}

The visual inspection of the first slide from the cascade impactor showed a good deal of coarse material other than the spores of micro-organisms. This material included fragments of plants, plant hairs, cell walls, faecal pellets of mites (packed with mould spores), cast skins of mites, as well as whole conidial heads of Aspergillus and sporangia of Mucoraceae (the last help to substantiate identification of the Mucoraceae spore type among the finer fractions). Few of these particles penetrated beyond the first stage of the impactor and material deposited in the later stages was predominantly microbial spores. The better hays seemed to have more non-spore dust than the mouldy hays, possibly because the process of becoming mouldy tended to bind the loose particles in a weft of mycelium. Plate 1 shows typical fields of cascade impactor slides of a good hay (G) and a farmer's lung hay (H 44).

\section{Possibility of penetration of particles into lungs}

The visual and cultural methods of assessing potential alveolar penetration in general agreed. For the two methods, Fig. 3 shows graphically the $\%$ of particles retained by the four cascade impactor slides and the six Andersen sampler plates. The values obtained are combined averages of results from mouldy and farmer's lung hays (groups $c, d$ ). According to visual estimates, only $8 \%$ of spores of $\mathrm{Humi}$ - 
cola lanuginosa and the Mucoraceae penetrated beyond stage 2 of the cascade impactor, but $43 \%$ of Aspergillus and $55 \%$ of actinomycete spores were 'penetrating', by the criteria mentioned under Methods. According to cultural tests, however, a larger proportion of spores were 'penetrating', namely, $60 \%$ of the Mucoraceae and over $\mathbf{8 0} \%$ of the other groups. This discrepancy is partly attributed to the fact that the first two plates received clumps of spores (or even whole sporangia as in stage 1 of Mucoraceae) which, although they contained many spores, produced only one colony. Further, discrimination between single spores of Mucoraceae and members of the Aspergillus-Penicillium type was sometimes difficult; although spores of Absidia spp. are easily recognized, some of Mucor pusillus may have been misclassified, suggesting that the Mucoraceae types (Fig. 3) appeared to be less

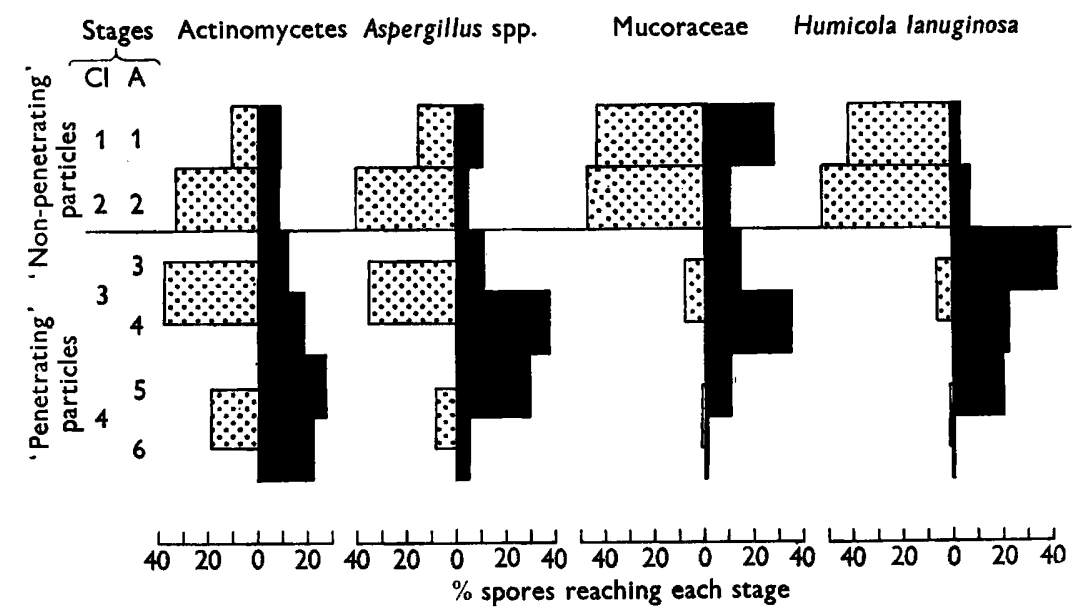

Fig. 3. Percentage penetration of spores to the four stages of the cascade impactor (CI; dotted), and to the six stages of the Andersen sampler (A; solid), average of groups $C$ and $d$.

penetrating than they actually were. Spores of Humicola lanuginosa are about $9 \mu$ diam. and the maximum proportion of $42 \%$ were found on stage 3 ; those of Aspergillus are about $4 \mu$ and the maximum of $38 \%$ was found on stage 4 , but $30 \%$ penetrated as far as stage 5. Hemispora stellata has smaller spores, most were found on stage 5 and hardly any on stage 6. Spores of the actinomycetes penetrated deeply, $27 \%$ reaching stage 5, and $23 \%$ stage 6 . Evidently many single spores of fungi and the majority of actinomycete spores could penetrate to the alveoli.

\section{DISCUSSION}

Our standard method of examining hays gives results that are reproducible enough to characterize batches of hay. Standardizing the humidity of the air in the wind tunnel would be a desirable improvement, as Zoberi (1961) found that humidity greatly affected the numbers of dry spores liberated into wind; but since a bale of hay is a concretion of micro-environments, errors from differences between bales and between parts of a single bale (Table 1) are likely to outweigh the humidity factor. Because the dust load is so easily shaken out, it was not considered feasible to work with a subsample taken after thoroughly mixing several separate samples. 
Incidental to the main object of the work, the Andersen sampler proved an excellent tool for isolating thermophilic actinomycetes from natural substrata. Dilution in air, instead of in water by the traditional manner, and impacting the spores from suspension in air directly on to the previously dried surface of halfstrength nutrient agar containing actidione, prevented contamination by bacteria and moulds, and on incubation overnight at $60^{\circ}$ gave numerous colonies of thermophilic actinomycetes (Gregory \& Lacey, 1962).

The proportion of spores seen on cascade impactor slides which was actually obtained in culture with the Andersen sampler was always small, and averaged about one in a thousand, even after allowing for the fact that two-thirds of the spore load had been lost from the sample during the $3 \mathrm{~min}$. blowing period for the cascade impactor run before the $\mathbf{1 5}$ sec. run with the Andersen sampler started (Table 8). Reasons for this small \% recovery in culture include the following: (1) no correction was applied for multiple infection, so that the counts tended to be gross underestimates, especially when nearly the maximum number of 400 colonies are recorded on one plate; (2) the media, aeration conditions and isolation temperatures may have been unsuitable for some organisms; (3) slow-growing organisms may have been overgrown or antagonized on the crowded plates; (4) many spores may have been dead. Table 8 shows that the percentage obtained in culture was larger with the better-quality hays. From these tests it is clear that both the mouldy and farmer's lung hays (groups $c$ and $d$ ) are important natural reservoirs of several moulds reported to be pathogenic to man or animals, e.g. Absidia ramosa and A. corymbifera, Aspergillus fumigatus, Hemispora stellata, Mucor pusillus, Scopulariopsis brevicaulis and Stachybotrys sp.

Table 8. Numbers obtained in culture on Andersen sampler plates as percentage (\%) of spores recorded visually on cascade impactor slides

$\begin{array}{lll}\text { Hays } & \text { Moulds } & \begin{array}{c}\text { Actinomycetes } \\ \text { and bacteria }\end{array} \\ \text { Group } b & 0.34 & \begin{array}{l}\mathbf{0 . 2 2} \\ \text { Group } c\end{array} \\ \text { Group } d & \mathbf{0 . 1 0} & \mathbf{0 . 1 1} \\ 0.03\end{array}$

The tests show it possible to characterize farmer's lung hays (group $d$ ). They had clearly heated spontaneously and become mouldy during maturation, and tended to be neutral in contrast to the low $\mathrm{pH}$ values of good hays or other mouldy hays examined. They had many more thermophilic organisms, including Aspergillus fumigatus, and Humicola lanuginosa, than other mouldy hays, and were rich in thermophilic actinomycetes (especially at $60^{\circ}$ ), which were often seen microscopically to be growing vigorously on the hay and on mould hyphae and spores. Several hays of the mouldy group $c$ might well have been classified as farmer's lung hays had they come into contact with a susceptible patient. In spite of their abundance, and evidence of their potential ability to penetrate to the alveoli, it is uncertain whether any of these organisms play any direct part in the aetiology of farmer's lung disease, but the possibility that the actinomycetes are active merits serious attention in view of the occurrence of the disease after inhalation of white dust from mouldy hay reported by Campbell (1932) and Frank (1958, p. 202). 
We thank the Agricultural Research Council, at whose request this study was undertaken, for a grant to start the work; also Mr P. K. C. Austwick (Central Veterinary Laboratory, Weybridge) for help throughout the work. For batches of hay to test we are indebted to the following: Mr D. E. Amesbury (Newbury), Dr T. A. Blyton (Chesterfield), Dr D. O. Clarke (Weston-Super-Mare), Dr A. W. Frankland (St Mary's Hospital, Paddington), Dr C. J. Fuller (Exeter), Mr M. Jenkins (Brampton), Dr S. T. H. Jenkins (Aberystwyth), Messrs F. S. Mitchell and G. Shepperson (N.I.A.E., Silsoe), Mr L. Ogilvie (N.A.A.S., Bristol), Mr J. Sage (Honiton), Dr R. M. E. Seal (Penarth), Mr L. Thomas (Knighton), Mr J. W. Thwaite (Liskeard), and Dr J. McA. Williams (Hartington). We also thank Messrs V. Stansfield and F. Cowland for photography, and Miss M. Goodson for technical assistance.

\section{REFERENCES}

ANDERsen, A. A. (1958). New sampler for the collection, sizing, and enumeration of viable airborne particles. J. Bact. 76, 471 .

Brierley, W. B., Jewson, S. T. \& Brierley, M. (1927). The quantitative study of soil fungi. Proc. Int. Congr. Soil Sci., Washington, 3, 48.

Browne, C. A. (1929). The spontaneous combustion of hay. U.S.D.A. Technical Bull. no. 141, 38 pp.

Camprelt, J. M. (1932). Acute symptoms following work with hay. Brit. med. J. 2, 1143.

Davies, C. N. (1952). Dust sampling and lung disease. Brit. J. Industr. Med. 9, 120.

EAstwood, D. J. (1948). The fungus flora of composts. Trans. Brit. mycol. Soc. 35, 215.

Forsyth, W. G. C. \& Webley, D. M. (1948). The microbiology of composting. II. A study of the aerobic thermophilic bacterial flora developing in grass composts. Proc. Soc. appl. Bact. 11, 34.

Frank, R. C. (1958). Farmer's lung-a form of pneumoconiosis due to organic dusts. Amer. J. Roentgenol. 79, 189.

Fulder, C. J. (1958). Farmer's lung; in Fungous Diseases and Their Treatment, p. 138. Ed. R. W. Ridell \& G. T. Stewart, London: Butterworth.

Gregory, P. H. \& LACEY, M. E. (1962). The isolation of thermophilic actinomycetes. Nature, Lond. 195, 95.

Gregory, P. H. \& LACEY, M. E. (1963). Liberation of spores from mouldy hay. Trans. Brit. mycol. Soc. (in the Press).

MAY, K. R. (1945). The cascade impactor: an instrument for sampling coarse aerosols. J. Sci. Instrum. 22, 187.

Miene, H. (1930). Utber die Selbsterhitzung des Heues. (2. Aufl.) Arb.dtsch. LandwG. 196, 1.

Truninger, E. (1929). Allgemeine Beobachtungen und Untersuchungen über Wesen, Ursache und Verlauf der Selbsterhitzung und Selbstentzündung von Dürrfutter. Landre. Jb. Schroeiz, 43, 278.

Webley, D. M. (1947). The microbiology of composting. I. The behaviour of the aerobic mesophilic bacterial flora of composts and its relation to other changes taking place during composting. Proc. Soc. appl. Bact. 10, 83.

ZoBERI, M. H. (1961). Take-off of mould spores in relation to wind speed and humidity. Ann. Bot., Lond. 25, 53.

\section{EXPLANATION OF PLATE 1}

Photomicrographs $(\times 600)$ from cascade impactor slides $1,2,3$ and 4 to compare a good hay with a farmer's lung hay.

$a$ Farmer's lung hay from Devon (H 44).

$b$ Good hay from Rothamsted Experimental Farm (G, 1961). 

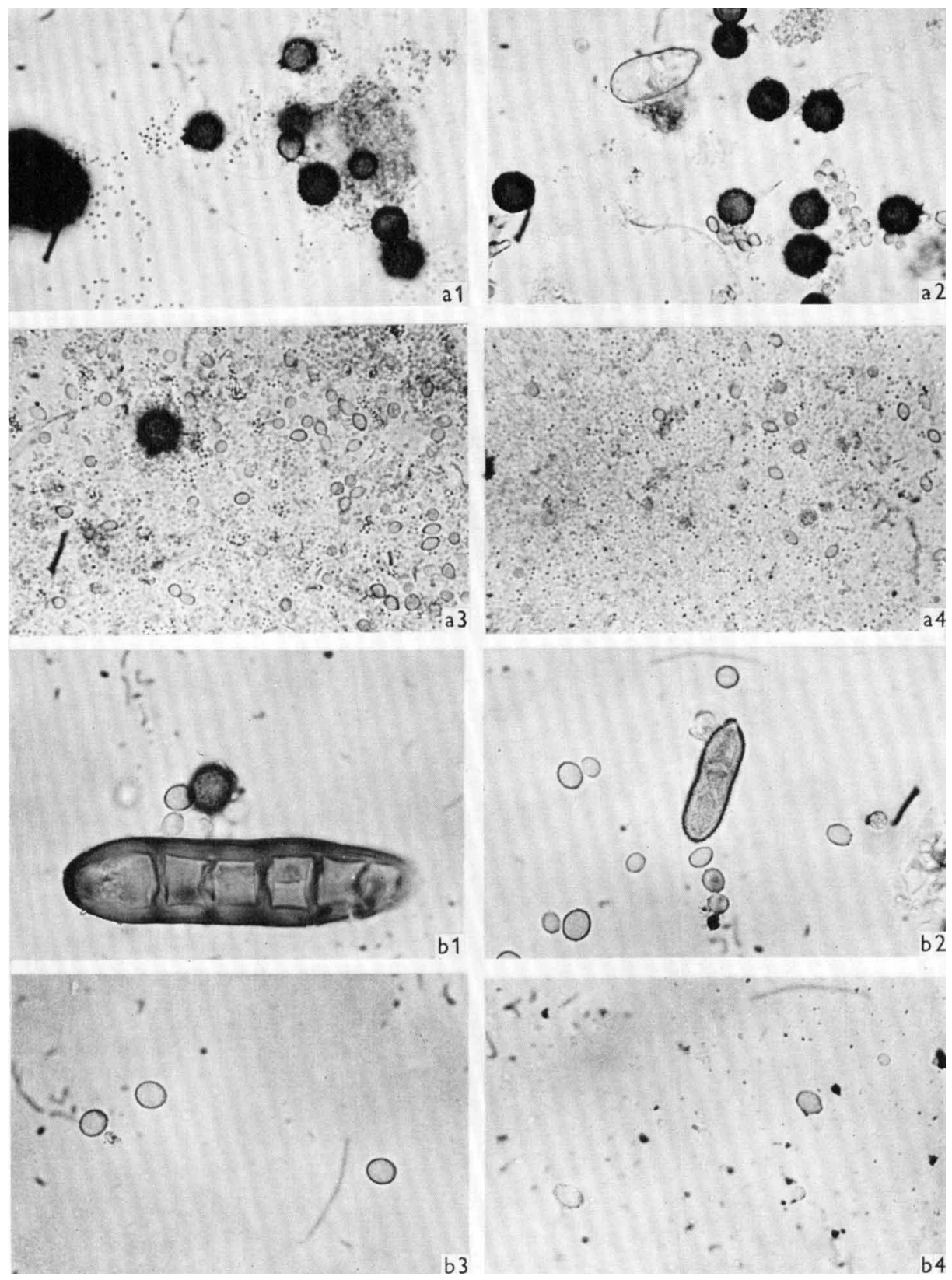

P. H. GREGORY AND M. E. LACEY

(Facing $p .88$ ) 